\title{
Lipid Management Strategies for the Prevention of Adverse Cardiovascular Events
}

\author{
Patrice Delafontaine Brian C. Weiford \\ Division of Cardiovascular Diseases, The University of Kansas Medical Center, Kansas City, Kans., USA
}

As the leading cause of death and a tremendous source of morbidity worldwide [1], atherosclerotic coronary artery disease (CAD) and its treatment and prevention represent one of the greatest challenges facing health care. Aggressive efforts at educating the public about CAD and identifying high-risk individuals as part of primary prevention of CAD are needed. Risk factor modification, including lipid lowering, remains a cornerstone of prevention and treatment of CAD. Statin drugs represent a highly effective and well-tolerated option in the effort to treat dyslipidemia. While many patients may achieve the lipid profile goals outlined by the National Cholesterol Education Program Adult Treatment Panel III (ATP III) with statin therapy alone, some require the addition of further pharmacologic agents to control abnormal lipids and reduce the incidence of cardiovascular events. In a review of statins as monotherapy and as part of combination treatment, Carmena [2] highlights current and future options for lipid-lowering agents as well as their efficacy and safety profiles.

The identification of individuals at high risk for the development of CAD and adverse cardiovascular events who would then benefit from intensified lipid-lowering efforts is a primary focus of the most recent guidelines of the ATP III [3]. Patients with the metabolic syndrome, marked by insulin resistance, truncal obesity, impaired glucose tolerance and dyslipidemia, including high triglycerides and low high-density lipoprotein (HDL) levels, represent a subset at very high risk. These individuals may have a $20 \%$ or greater risk of developing CAD within 10 years according to the Framingham absolute risk estimate [4], and aggressive modification of their dyslipidemia as part of a comprehensive risk factor reduction plan is in order. Another new feature of the ATP III guidelines is the lowering of the 'low' HDL cutoff point from $<35$ to $<40 \mathrm{mg} / \mathrm{dl}$. The impact of treating low HDL was illustrated in the Lipoprotein and Coronary Atherosclerosis Study, in which statin-treated patients with low HDL experienced the greatest reduction in angiographic progression of CAD and adverse cardiovascular events [4]. However, because of the less robust effects of statins in raising HDL compared to their low-density lipoprotein (LDL)-lowering effects, patients with mixed dyslipidemias may not reach treatment goals with statin monotherapy.

Reluctance to utilize combination therapy with statins and niacin has stemmed from concerns about myopathy and hepatotoxicity reported with lovastatin and niacin in combination shortly after lovastatin was introduced in 1987 [5]. As Guyton and Capuzzi [5] point out in a review

\section{KARGER \\ Fax +4161306 1234 \\ E-Mail karger@karger.ch \\ www. karger.com

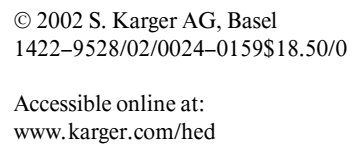

Dr. Patrice Delafontaine

Division of Cardiology, Kansas University Medical Center

3901 Rainbow Boulevard

Kansas City, KS 66160-7378 (USA)

Tel. +1 913588 6015, Fax +1 913588 6010, E-Mail pdelafontaine@kumc.edu 
of trials reported between 1994 and 1997 using combination statin-niacin therapy with $>400$ patients, the combination can be effective and safe when careful attention is paid to close monitoring, patient education, using lower dosages of both drugs and the particular niacin formulation. Cases of significant hepatic and muscle toxicity have been reported in patients using high doses of statin and niacin, and significant transaminase elevations have been observed in patients treated with the combination of a statin and a sustained-release form of niacin given twice daily [5].

In a recently reported study of 160 patients with established CAD, normal LDL and low HDL followed for 3 years [6], the combination of simvastatin and niacin was shown to increase HDL cholesterol by $26 \%$, reduce LDL cholesterol by $42 \%$ and achieve a statistically significant reduction in proximal coronary artery stenoses compared to placebo. The rate of major adverse cardiovascular events was significantly reduced as well, with a $90 \%$ reduction in the risk of cardiovascular death, myocardial infarction, stroke or revascularization compared to placebo. An abstract recently presented at the American College of Cardiology Scientific Sessions [7] highlighted the safe and efficacious use of a single-tablet extended-release niacin-lovastatin combination as compared to simvastatin or atorvastatin alone. Similar reductions in LDL cholesterol were observed, with significant improvement in HDL cholesterol (+19 vs. +3 and $+8 \%)$ and triglyceride lowering (32 vs. 23 and 6\%), when extended-release niacin $1,000 \mathrm{mg}$ plus lovastatin $40 \mathrm{mg}$ was compared to atorvastatin $20 \mathrm{mg}$ and simvastatin $20 \mathrm{mg}$. No myopathy or significant hepatotoxicity was reported with combination therapy.

Similar concerns about myopathy have limited the use of combinations of statins and fibrates for the treatment of mixed dyslipidemias. Twelve cases of myopathic reactions, including rhabdomyolysis and renal failure, were reported from 1987 to 1988 with the use of a combination of lovastatin and gemfibrozil [8]. However, as Carmena [2] points out, subsequent review of multiple studies with $>1,600$ patients examining the safety and efficacy of statin-fibrate combination therapy has not shown any cases of rhabdomyolysis or renal failure and a much lower incidence of myopathy $(0.12 \%)$ than early studies suggested.

The primary lipid therapy target for patients with risk factors for CAD or established CAD remains lowering levels of LDL cholesterol. In those patients unable to achieve target LDL cholesterol levels with maximal (or maximally tolerated) doses of statins, newer agents, including colesevelam, plant sterols and ezetimibe, appear to be effective and safe adjuncts to further lower LDL [2]. Several studies recently presented at the American College of Cardiology Scientific Sessions investigating the efficacy of ezetimibe in combination with statin drugs showed significantly reduced LDL levels compared to statin monotherapy, with safety profiles similar to statins alone or placebo [9-11]. Newer, more potent statins may be beneficial in achieving ATP III LDL cholesterol goals at lower doses while having a more substantial effect on raising HDL. In a study comparing efficacy at starting doses of rosuvastatin compared to atorvastatin [12], rosuvastatin showed statistically significantly greater reductions in LDL compared to atorvastatin and a higher percentage of patients with baseline LDL of $160-250 \mathrm{mg} / \mathrm{dl}$ who achieved the LDL treatment goal. In addition, a 50\% greater increase in HDL cholesterol was noted with rosuvastatin compared to atorvastatin and a safety profile similar to atorvastatin and placebo.

Despite the availability of many effective and relatively safe agents for the treatment of dyslipidemia, there is still room to optimize abnormal lipid parameters in atrisk patients in order to reduce adverse cardiovascular events. As evidenced by the Lipid Treatment Assessment Project [13], a significant proportion of high-risk patients and those with established CAD are not achieving target LDL cholesterol levels with current therapies. Of nearly 5,000 patients with dyslipidemia stratified into risk categories for CAD or established CAD, only 38\% reached target National Cholesterol Education Program levels, and success rates in achieving target levels were lowest in those with known CAD. Similar success rates in the treatment of dyslipidemic patients in Europe have been shown [2]. Such findings underscore the need for more aggressive treatment of dyslipidemia and the identification of those at high risk for cardiovascular morbidity who would most benefit from such treatment. 


\section{References}

1 Knopp RH: Drug treatment of lipid disorders. N Engl J Med 1999;341:498-511.

2 Carmena R: Statins: Monotherapy or combination strategy for the treatment of dyslipidemia. Heart Drug 2002;2:175-183.

3 Executive Summary of The Third Report of The National Cholesterol Education Program (NCEP) Expert Panel on Detection, Evaluation, and Treatment of High Blood Cholestero in Adults (Adult Treatment Panel III). JAMA 2001;285:2486-2497.

4 Ballantyne CM: Treating mixed dyslipidemias: Why and how. Clin Cardiol 2001;24(7 suppl): II-6-II-9.

5 Guyton J, Capuzzi D: Treatment of hyperlipidemia with combined niacin-statin regimens. Am J Cardiol 1998;82:82U-86U.
6 Brown BG, Zhao XQ, Chait A, Fisher LD, Cheung MC, Morse JS, Dowdy AA, Marino EK, Bolson EL, Alaupovic P, Frohlich J, Albers $\mathrm{JJ}$ : Simvastatin and niacin, antioxidant vitamins, or the combination for the prevention of coronary artery disease. N Engl J Med 2001; 345:1583-1592.

7 Bays H: Lipoprotein effects of a new dual component drug (extended-release niacin/lovastatin) compared to starting doses of atorvastatin and simvastatin. J Am Coll Cardiol 2002; 39(suppl A):245A.

8 Shepherd J: Fibrates and statins in the treatment of hyperlipidaemia: An appraisal of their efficacy and safety. Eur Heart J 1995;16:5-13.

9 Davidson M, et al: Ezetimibe co-administered with simvastatin in 668 patients with primary hypercholesterolemia. J Am Coll Cardiol 2002; 39(suppl A):226A.

10 Ballantyne C, et al: Ezetimibe co-administered with atorvastatin in 628 patients with primary hypercholesterolemia. J Am Coll Cardiol 2002; 39(suppl A):227A.
11 Bays H, et al: Ezetimibe added to ongoing statin therapy for treatment of primary hypercholesterolemia - administered with simvastatin in 668 patients with primary hypercholesterolemia. J Am Coll Cardiol 2002;39(suppl A): 245A.

12 Davidson M, Ma P, Stein EA, Gotto AM Jr, Raza A, Chitra R, Hutchinson H: Comparison of effects on low-density lipoprotein cholesterol and high-density cholesterol with rosuvastatin versus atorvastatin in patients with type IIa or IIb hypercholesterolemia. Am J Cardiol 2002; 89:268-275.

13 Pearson TA, Laurora I, Chu H, Kafonek S: The lipid treatment assessment project (L-TAP): A multicenter survey to evaluate the percentages of dyslipidemic patients receiving lipid-lowering therapy and achieving low-density lipoprotein cholesterol goals. Arch Intern Med 2000; 160:459-467. 